EFOMP policy statement

\title{
European Federation of Organisations for Medical Physics (EFOMP) Policy Statement 12.1: Recommendations on Medical Physics Education and Training in Europe 2014
}

\author{
C.J. Caruana ${ }^{\text {a, }}$, S. Christofides ${ }^{\text {b }}$, G.H. Hartmann ${ }^{c}$ \\ ${ }^{a}$ Medical Physics Department, Faculty of Health Sciences, University of Malta, Malta \\ ${ }^{\mathrm{b}}$ Medical Physics Department, Nicosia General Hospital, Cyprus \\ ${ }^{\mathrm{c}}$ Department of Medical Physics and Radiation Therapy, German Cancer Research Centre in Heidelberg, Germany
}

\section{A R T I C L E I N F O}

\section{Article history:}

Available online 20 June 2014

\section{Keywords:}

EFOMP

Medical Physicist

Medical Physics Expert

Education and training

Qualification and curriculum frameworks

\begin{abstract}
A B S T R A C T
In 2010, EFOMP issued Policy Statement No. 12: "The present status of Medical Physics Education and Training in Europe. New perspectives and EFOMP recommendations" to be applied to education and training in Medical Physics within the context of the developments in the European Higher Education Area arising from the Bologna Declaration and with a view to facilitate the free movement of Medical Physics professionals within Europe. Concurrently, new recommendations regarding qualifications frameworks were published by the European Parliament and Council which introduced new terminology and a new qualifications framework - the European Qualifications Framework (EQF) for lifelong learning. In addition, a new European directive involving the medical use of ionizing radiations and set to replace previous directives in this area was in the process of development. This has now been realized as Council Directive 2013/59/Euratom of 5 December 2013 which has repealed directive 97/43/Euratom. In this regard, a new document was developed in the context of the EC financed project "European Guidelines on the Medical Physics Expert" and published as RP174. Among other items, these guidelines refer to the mission statement, key activities, qualification framework and curricula for the specialty areas of Medical Physics relating to radiological devices and protection from ionizing radiation. These developments have made necessary an update of PS12; this policy statement provides the necessary update.
\end{abstract}

๑ 2014 Published by Elsevier Ltd on behalf of Associazione Italiana di Fisica Medica.

\section{Introduction}

In 2010, EFOMP issued Policy Statement No. 12: "The present status of Medical Physics Education and Training in Europe. New perspectives and EFOMP recommendations" to be applied to education and training in Medical Physics within the context of the developments in the European Higher Education Area arising from the Bologna Declaration and with a view to facilitate the free movement of Medical Physics professionals within Europe [1]. Concurrently, new recommendations regarding qualifications frameworks were published by the European Parliament and Council which introduced new terminology and a new qualifications framework - the European Qualifications Framework (EQF) for

\footnotetext{
* Corresponding author. Tel.: +356 99486920.

E-mail address: carmel.j.caruana@um.edu.mt (C.J. Caruana).
}

lifelong learning [2]. In addition, a new European directive involving the medical use of ionizing radiations and set to replace previous directives in this area was in the process of development. This has now been realized as Council Directive 2013/59/Euratom of 5 December 2013 which has repealed directive 97/43/Euratom among others [3]. A new document of direct interest to the Medical Physics profession was therefore developed in the context of the EC financed project "European Guidelines on the Medical Physics Expert", and published as RP174 [4]. This important document will be referred to as the "Guidelines" in this policy statement. Among other items, these Guidelines refer to the mission statement, key activities, qualification framework and curricula in the specialty areas of Medical Physics relating to radiological devices and protection from ionizing radiation, namely Diagnostic and Interventional Radiology (D\&IR), Radiation Oncology (RO) and Nuclear Medicine (NM). All these developments have made necessary an update of PS12; this policy statement provides the necessary update. 


\section{Main elements of the guidelines with a direct impact on the present EFOMP policy statement}

The main elements of the guidelines with a direct impact on the present EFOMP policy statement are the mission statement, key activities, qualification framework and curriculum framework. This policy statement assimilates these four elements as professional qualification and curriculum frameworks should follow mission and role. The policy statement also generalizes these four elements to make them applicable to all specialties of Medical Physics, that is to not only those specialties of Medical Physics involving radiological medical devices and ionizing radiation (addressed in the Guidelines) but also to those specialties involving non-radiological medical devices (such as physiological measurement, neurology, audiology) and protection from other physical agents (such as strong electromagnetic static and RF fields, ultrasound, optical radiation, vibration) [3,5-14]. The four elements will be discussed in turn. In those states where the scope of the roles of the Medical Physicist (MP) and Medical Physics Expert (MPE) are presently delimited to radiological medical devices and ionizing radiation, the policy statement may if desired be amended by replacing 'medical devices' with 'radiological medical devices' and 'physical agents' by 'ionizing radiations'.

\section{Mission statement for Medical Physicists and Medical Physics Experts}

The new mission statement for medical physicists and medical physics experts reads as follows: "Medical Physicists and Medical Physics Experts will contribute to maintaining and improving the quality, safety and cost-effectiveness of healthcare services through patient-oriented activities requiring expert action, involvement or advice regarding the specification, selection, acceptance testing, commissioning, quality assurance/control and optimized clinical use of medical devices and regarding patient risks from associated physical agents including protection from such physical agents, installation design and surveillance, and the prevention of unintended or accidental exposures to physical agents; all activities will be based on current best evidence or own scientific research when the available evidence is not sufficient. The scope includes risks to

Table 1

The key activities of the Medical Physicist and Medical Physics Expert.

\begin{tabular}{l}
\hline Key activity \\
\hline Scientific problem solving service. \\
Dosimetry measurements (all physical agents \\
e.g., effective dose in ionizing radiation, \\
SAR in MRI, thermal and mechanical \\
indices in ultrasound). \\
Patient safety/risk management (including \\
volunteers in biomedical research, carers, \\
comforters and persons subjected to \\
non-medical procedures using \\
medical devices). \\
Occupational and public safety/risk \\
management when there is an impact \\
on medical exposure or own safety
\end{tabular}

Clinical medical device management.

Clinical involvement.

Development of service quality and cost-effectiveness.

Expert consultancy.

Education of healthcare professionals

(including medical physics trainees)

Health technology assessment (HTA)

Innovation

Main actions

Comprehensive scientific problem solving service involving recognition of less than optimal performance or optimized use of medical devices, identification and elimination of possible causes or misuse, and confirmation that proposed solutions have restored device performance and use to acceptable status. All activities are to be based on current best scientific evidence or own research when the available evidence is not sufficient.

Measurement and calculations of dose received by patients, volunteers in biomedical research, carers, comforters and persons subjected to non-medical procedures using medical devices for the purpose of supporting justification and optimization processes; selection, calibration and maintenance of dosimetry related instrumentation; independent checking of dose provided by dose reporting devices (including software devices); measurement of quantities required as inputs to dose reporting or estimating devices (including software). Measurements to be based on current recommended techniques and protocols. Surveillance of medical devices and evaluation of clinical protocols to ensure the on-going protection of patients, volunteers in biomedical research, carers, comforters and persons subjected to non-medical procedures using medical devices from the deleterious effects of physical agents in accordance with the latest published evidence or own research when the available evidence is not sufficient. Includes optimization, the development of risk assessment protocols, including the analysis of events involving, or potentially involving, accidental or unintended medical exposures to physical agents and dose audit. Surveillance of medical devices and evaluation of clinical protocols with respect to the protection of workers and public from physical agents when impacting the exposure of patients, volunteers in biomedical research, carers, comforters and persons subjected to non-medical procedures or responsibility with respect to own safety. Correlation of occupational, public and medical

exposures - balancing occupational and public risk and patient needs.

Provide technical advice and participate in the specification, selection, acceptance testing, commissioning, installation design and decommissioning of medical devices in accordance with the latest published European or International recommendations. The specification, management and supervision of associated quality assurance/control programmes. Design of all testing protocols is to be based on current European or international recommended techniques and protocols.

Carrying out, participating in and supervising everyday patient physical agent protection and quality control procedures to ensure on-going effective and optimized use of medical devices and including patient specific optimization, prevention of unintended or accidental exposures and patient follow-up. Optimization of protocols before first use with patients via the use of anthropomorphic phantoms and simulation using specialized physical agent dosimetry software.

Support the introduction of new medical devices into clinical service, lead the introduction of new medical physics services and participate in the introduction/development of clinical protocols/techniques whilst giving due attention to economic issues.

Provision of expert advice to outside clients (e.g., smaller clinics with no in-house medical physics expertise). Contributing to quality healthcare professional education through knowledge transfer activities concerning the technical-scientific knowledge, skills and competences supporting the clinically-effective, safe, evidence-based and economical use of medical devices. Participation in the education of medical physics students and organization of medical physics residency programmes.

Taking responsibility for the physics component of health technology assessments related to medical devices and/or the medical uses of physical agents.

Developing new or modifying existing devices (including software) and improved use of protocols for the solution of hitherto unresolved clinical problems.

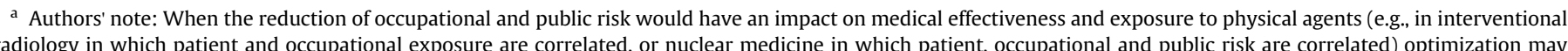

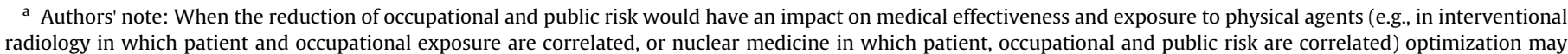

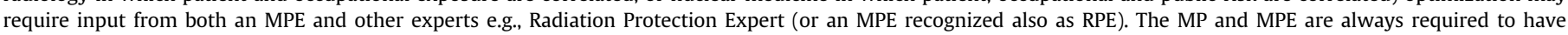
knowledge and skills in occupational radiation protection sufficient to take responsibility for own protection.
} 
volunteers in biomedical research and carers and comforters" [15-18]. The purpose for the formulation of this mission statement is to make the role of the MP and MPE more accessible to policy makers and the management of healthcare organizations.

\section{Key activities of Medical Physicists and Medical Physics Experts}

This mission is expressed in many aspects of medical physics practice. Definitions of the key activities of medical physicists and medical physics experts are shown in Table 1.

\section{Qualification framework for Medical Physicists and Medical Physics Experts in Europe}

The development of the qualification framework for the MP and MPE is guided by the following principles:

(a) All qualification frameworks in Europe should be referred to the EQF and the qualification framework for the MP and MPE is based on the levels defined by the EQF. For the purpose of this policy statement the appropriate levels are EQF Level 6 (e.g., Bachelor or equivalent), EQF Level 7 (e.g., Masters or equivalent) and EQF Level 8 (Expert level, highest level of the $\mathrm{EQF})$. The definition of these levels can be found in the EQF. Henceforth the MPE is defined as a clinically qualified MP who has reached EQF level 8 in his/her own specialty of clinical Medical Physics (e.g., Diagnostic and Interventional Radiology, Radiation Oncology, Nuclear Medicine, Physiological Measurement, Neurology, Audiology). (b) The qualification framework would make it possible for more individuals to achieve clinically qualified MP and MPE status through its flexibility, cost-effectiveness and lifelong learning approach.

(c) The qualification framework would facilitate the mobility of the clinically qualified MP and MPE in Europe through an agreed set of minimum criteria for achievement of such status.

(d) Owing to the rapid expansion of medical device technology and physical agent research publication, it is becoming increasingly difficult for a MP and MPE to become competent in more than one specialty of medical physics; therefore, early specialization has become a necessity and the MP and MPE should be independently recognized in each specialty of medical physics [19-21].

The qualification framework (Fig. 1) is based on the results of a survey carried out during the 'European Guidelines on the Medical Physics Expert' project, the various systems of qualifications used in Europe and the best features of national systems taking into account the modernization of scientific careers envisaged in the field. The knowledge, skills and competences (KSC) for the recognition of MP and MPE status are to be gained initially through learning in an institution of higher education and structured clinical training in a residency within an accredited healthcare institution to gain recognition as a clinically qualified MP (EQF Level 7+) and subsequently developed to expert level (EQF level 8) through further structured advanced experience and CPD to gain recognition as a MPE by competent authorities (or the equivalent expert level in the fields of medical devices and physical agents other than radiological devices and ionizing radiation). Explanatory notes to the qualification framework diagram plus associated rationales are shown in Table 2.

\section{Qualification Framework for the Medical Physicist and Medical Physics Expert in Europe}

The Qualifications Framework is based on the European Qualifications Framework (EQF). In the EQF learning outcomes are defined in terms of Knowledge, Skills, Competences (KSC) (European Parliament and Council 2008/C 111/01)

\begin{tabular}{|c|c|}
\hline \multicolumn{2}{|c|}{ EDUCATION } \\
\hline EQF Level 6 & EQF Level 7 \\
$\begin{array}{c}\text { (e.g., Bachelor } \\
\text { with } 180-240\end{array}$ & $\begin{array}{c}\text { (e.g., Master } \\
\text { with } 90-120\end{array}$ \\
\hline ECTS) & ECTS) \\
(i) & (iii) \\
\hline Physics & Medical \\
or & Physics* \\
equivalent & or \\
(ii) & equivalent
\end{tabular}

(iv)

CLINICAL TRAINING
Certification
as Medical Physicist
in a given specialty
(v)
Structured accredited
clinical training
residency in the
specialty of Medical
Physics for which the
candidate seeks
clinical certification.
The duration should
be typically two full-
time year
equivalents**

(vi)

ADVANCED
EXPERIENCE and CPD
EQF Level 8 in
Medical Physics
specialty

(vii)

Structured advanced experience and CPD in the specialty of Medical Physics for which the candidate seeks certification as MPE.

The duration would be a further minimum additional two full-time year equivalents*** (viii)

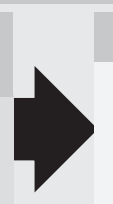

RECOGNITION

Certification as Medical Physics Expert in Medical Physics specialty (ix)

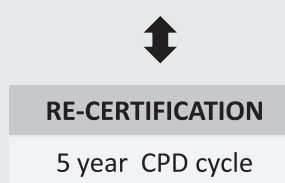

$(x)$

* Should include as a minimum the educational components of the Core KSC of Medical Physics and the educational components of the KSC of the Specialty of Medical Physics (e.g., Diagnostic \& Interventional Radiology, Nuclear Medicine, Radiation Oncology, Physiological Measurement) for which the candidate seeks clinical certification. When this element of specialization is not included it must be include $d$ in the residency. ** The EQF level of the residency is intermediate between EQF levels 7 and 8 . The training can follow or be concurrent with the Masters. *** In countries where the MPE is required to be certified in further specialties of Medical Physics the number of years would need to be extended such that the MPE will achieve EQF level 8 in each specialty. 
Table 2

Notes to the qualification framework diagram.

\begin{tabular}{|c|c|c|}
\hline & Note & Rationale \\
\hline (i) & $\begin{array}{l}\text { The fundamental educational level for medical physics } \\
\text { professionals is a level } 6 \text { in physics and associated } \\
\text { mathematics [1] }\end{array}$ & $\begin{array}{l}\text { Medical Physics professionals need to have good foundations in physics and } \\
\text { mathematics as Medical Physics is a physical, numeric and exact science. }\end{array}$ \\
\hline (ii) & $\begin{array}{l}\text { 'Equivalent' here meaning EQF level } 6 \text { with a high level } \\
\text { of physics and mathematics content. }\end{array}$ & $\begin{array}{l}\text { This will make it possible for graduates from other Level } 6 \text { programmes which } \\
\text { include a high level of physics and mathematics (e.g., engineering, biophysics) } \\
\text { to enter the field. }\end{array}$ \\
\hline (iii) & $\begin{array}{l}\text { The educational entry level for the medical physics } \\
\text { professional has been set at EQF level } 7 .\end{array}$ & $\begin{array}{l}\text { At entry level the medical physics professional needs to have highly specialized } \\
\text { knowledge, critical awareness of knowledge issues in the field, specialized } \\
\text { problem-solving skills, ability to manage work contexts that are complex and } \\
\text { ability to review the performance of teams [2]. Medical physics professionals } \\
\text { require highly specialized knowledge in protection from physical agents and } \\
\text { medical devices and specialized problem-solving and troubleshooting skills. } \\
\text { The medical physics professional is involved in clinical contexts that may be } \\
\text { very complex and reviews the performance of physical agent protection and } \\
\text { quality control teams in own specialty of medical physics. }\end{array}$ \\
\hline (iv) & $\begin{array}{l}\text { 'Equivalent' here meaning EQF level } 7 \text { with a high level } \\
\text { of physics and mathematics content plus the educational } \\
\text { component of the core KSC of medical physics and the } \\
\text { educational component of the KSC specific to the specialty } \\
\text { of medical physics for which the candidate would be } \\
\text { seeking clinical certification. This additional education }\end{array}$ & $\begin{array}{l}\text { This will make it possible for candidates with Masters in physics, biophysics, } \\
\text { engineering etc. to enter the field; however, such candidates need to undertake } \\
\text { an additional educational programme which includes the educational component } \\
\text { of the core KSC of medical physics and the educational component of the KSC } \\
\text { specific to the specialty of medical physics for which the candidate would be } \\
\text { seeking clinical certification. }\end{array}$ \\
\hline
\end{tabular}

(v) The Medical Physicist at entry level is a professional with clinical certification in medical physics i.e., having a level of education in medical physics at a level intermediate between EQF levels 7 and 8, having typically 2 years full-time equivalent accredited clinical training and recognized as competent to act independently through enrollment in a national register for Medical Physicists.

(vi) Structured accredited residency based training for clinically based development of the core KSC of medical physics and the KSC specific to the specialty of medical physics for which the candidate would be seeking clinical certification.

The duration of this structured training is typically two full-time year equivalents.

(vii) The MPE in a given specialty of medical physics is a clinically qualified Medical Physicist with clinical certification in the specialty who has achieved the highest level of expertise in that particular specialty. The clinically qualified Medical Physicist through structured advanced experience, ongoing extensive CPD and commitment develops the KSC to the highest possible level i.e., EQF level 8.

The education and training to clinical certification in medical physics is a necessary foundation for further development to MPE EQF Level 8.

A training period of two full-time year equivalents for any one specialty of medical physics is recommended [19-21]

The qualification level for the MPE has been set at EQF Level 8 because the MPE requires knowledge at the most advanced frontier of a field of work and at the interface between fields, the most advanced and specialized skills and techniques, including synthesis and evaluation, required to solve critical problems in research/innovation and to extend/redefine existing professional practice, demonstrate substantial authority, innovation, autonomy, professional integrity and sustained commitment to the development of new ideas or processes at the forefront of work contexts including research [2]. To carry out activities requiring expert action, involvement or advice with authority and autonomy and which are based on current best evidence (or own scientific research when the available evidence is not sufficient), the MPE requires frontier knowledge in own specialty of medical physics and at the interface between physics and medicine. The MPE requires specialized skills and techniques in protection from physical agents and comprehensive experience regarding the effective and safe use of the medical devices in own specialty, and the synthesis and evaluation skills required to solve critical problems in service development, research, innovation and the extension and redefinition of existing professional practice.

It should be emphasized that the further 2 years to reach MPE status must consist of advanced, structured experience and CPD and not simply CPD designed to maintain competence as Medical Physicist. The two years minimum advanced experience must be measured from the time when the advanced experience commences. The advanced experience and CPD might not follow immediately the 2 years of basic training if the candidate is not deemed to be sufficiently prepared. It is to be understood that senior MPEs practicing in large medical centers with a full range of devices would need more years of advanced experience than the 2 years minimum. On the other hand small facilities can be serviced by novice MPEs working under the guidance of a senior MPE.

(ix) A person who is currently recognized as an MPE and is in possession of the core KSC of medical physics and the KSC specific to the specialty for which recognition is sought should be deemed to satisfy the requirements for recognition as an MPE if they are currently on active duty as an MPE and are deemed to have reached EQF level 8.

(x) This is the requirement for an MPE to maintain recognition.

A five year cycle for re-certification (i.e., recognition as having maintained an EQF level 8 in the particular specialty of Medical Physics) is recommended. 


\section{Curriculum framework for Medical Physicist and Medical Physics Expert educational and training programmes in Europe}

The curriculum framework is based on the concept of learning outcomes expressed in terms of knowledge, skills and competence (KSC) as specified and defined in the EQF. The definitions of the three types of learning outcomes at the different EQF levels can also be found in the EQF. The curriculum framework was designed with a core medical physics KSC and specialty KSC structure. By emphasizing areas of commonality in the various specialties (within the core KSC) the framework makes it easier for MP and MPE in different specialties to cooperate in the interest of the patient and also makes it possible to avoid undue fragmentation of the profession. The new curriculum framework is shown in Fig. 2 and is expressed as a structured inventory of required KSC underpinning the key activities of the MP and MPE. In addition, the KSC are classified in two categories, Generic and Subject Specific skills as defined in the documents of the European Higher Education Area [22]:

(a) Generic skills consist of transferable skills which are expected of all professionals at a particular level of the EQF. In this case the relevant levels are level 7 [22] and level 8 [23].

(b) Subject specific KSC are specific to a profession. These are further classified into sub-categories as determined by the particular profession. In the case of Medical Physics the subcategories are those specified in Refs. [24,25], that is:

(i) Medical Physics core KSC: these KSC are expected of all MP/MPEs irrespective of their specialty:

- KSC for the MP/MPE as physical scientist: these are fundamental physics KSC expected of all physical scientists
- KSC for the MP/MPE as healthcare professional: these are KSC expected of all healthcare professionals

- KSC for the MP/MPE as expert in the clinical use of medical devices and protection from associated physical agents: these represent medical device and safety KSC required by all specialties of medical physics.

(ii) Medical Physics Specialty KSC: these KSC are highly specific to each specialty of medical physics

It is important to note that an MP and MPE from one specialty of medical physics who is required to assume specific responsibilities from another specialty may be certified to carry out those specific responsibilities following the acquisition of the corresponding KSC. Such cases may arise for example in a small nuclear medicine facility that requires its nuclear medicine MP and MPE to take responsibility for the management of quality control testing of the CT component of a PET/CT system.

The Generic and Core KSC of medical physics and the three Specialty KSC inventories for Diagnostic and Interventional Radiology, Nuclear Medicine and Radiation Oncology are given in Annex 1 of the Guidelines. Specialty KSC inventories for other specialties of Medical Physics (e.g., Physiological Measurement) still need to be developed at the European level, although they have been developed at national levels (UK, Finland, Netherlands). A candidate seeking recognition as an MP and MPE in a given specialty of medical physics should reach the corresponding level (level $7+$ of the EQF if a Medical Physicist and level 8 of the EQF if Medical Physics Expert) in the core KSC of medical physics and the KSC specific to that particular specialty.

The question arises which of these KSC are expected to be achieved by the Medical Physicist at the end of the two years equivalent clinical training following (or concurrent with) the

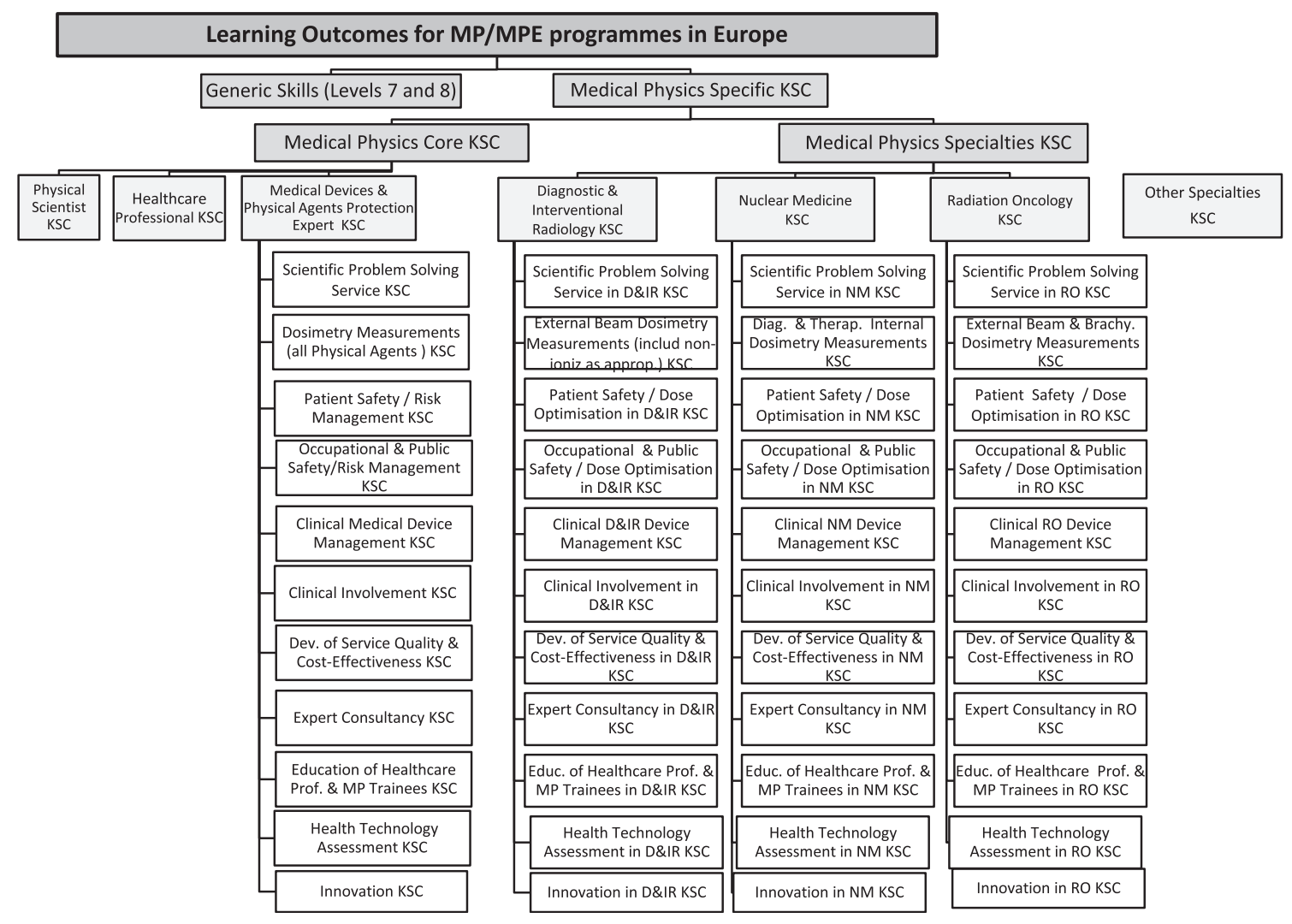

Figure 2. The curriculum framework for Medical Physics in Europe. 
Masters in Medical Physics (EQF level 7+) and which at the MPE level (EQF level 8)? In general most of the knowledge, a substantial number of the skills and some of the competences should be acquired by the end of the initial two year clinical training. However, as Medical Physics is by nature complex it must be emphasized that these skills and competences are developed over a period of years. The majority of the skills and competences would be acquired to a comprehensive degree only at the MPE level i.e., level 8. Educational and training methods should take into account modern developments in education and be based on approaches specific to adult learning (e.g., case-based learning). In the case of those specialties involving ionizing radiation, the skills and competences to be acquired by the end of the two years equivalent clinical training following (or concurrent with) the Masters in Medical Physics (EQF level 7+) are those defined by the following IAEA training documents [19-21]. Education and training programmes should be based on the most updated textbooks and reports in the literature. For example, in the case of those specialties involving ionizing radiation these would be:

a. Medical physics textbooks such as the handbooks and training manuals produced by the IAEA for physics in radiation oncology, nuclear medicine and diagnostic and Interventional radiology,

b. International, European and national legislation including all EU Directives relevant to radiation protection, medical devices, physical agents and personal protective equipment,

c. Relevant EC reports, recommendations and protocols (e.g., Radiation Protection Series http://ec.europa.eu/energy/nuclear/ radiation_protection/medical/publications_en.htm),

d. Reports, recommendations and protocols from relevant International organizations (e.g., IAEA, IEC, ICRP, ICRU, WHO, UNSCEAR),

e. Reports, recommendations and protocols from International, European and national medical physics professional bodies (e.g., IOMP, EFOMP, AAPM, IPEM),

f. Reports, recommendations and protocols from European professional and scientific bodies associated with the specific areas of medical physics practice (e.g., ESTRO, ECR, EANM),

g. Reports, recommendations and protocols from relevant national authorities (e.g., HPA (UK), STUK (FI))

h. Primary research reports and review articles from the research literature.

\section{Summary recommendation}

EFOMP strongly encourages its National Member Organizations to strive to ensure that the qualification frameworks and educational and training programmes in their respective states comply with the recommendations in this policy statement.

\section{Acknowledgments}

The authors would like to acknowledge all EFOMP Officers and others who have contributed to the final version of this document with comments and suggestions.

The EFOMP would like to thank the European Commission for providing the funds to make the 'European Guidelines on the Medical Physics Expert' project possible.

\section{References}

[1] Eudaldo T, Olsen K. European Federation of Organisations for Medical Physics. Policy Statement No.12: the present status of Medical Physics Education and Training in Europe. New Perspect EFOMP Recomm 2010;26(1):1-5.

[2] European Parliament and Council Recommendation 2008/C 111/01 on the establishment of the European Qualifications Framework for lifelong learning.

[3] EC. COUNCIL DIRECTIVE 2013/59/EURATOM of 5 December 2013 laying down basic safety standards for protection against the dangers arising from exposure to ionising radiation, and repealing Directives 89/618/Euratom, 90/641/ Euratom, 96/29/Euratom, 97/43/Euratom and 2003/122/Euratom.

[4] EC. (2014) RP174 European Guidelines on the Medical Physics Expert.

[5] EC Directive 2007/47/EC of the European Parliament and of the Council of 5 September 2007 amending Council Directive 90/385/EEC on the approximation of the laws of the Member States relating to active implantable medical devices, Council Directive 93/42/EEC concerning medical devices and Directive $98 / 8 / \mathrm{EC}$ concerning the placing of biocidal products on the market.

[6] EC. Directive 1998/79/EC of the European Parliament and of the Council of 27 October 1998 on in vitro diagnostic medical devices.

[7] EC. Directive 1993/42/EEC of 14 June 1993 concerning medical devices.

[8] EC. Directive 1990/385/EEC of 20 June 1990 on the approximation of the laws of the member states relating to active implantable medical devices.

[9] EC. 2013/35/EU of the European parliament and of the council of 26 June 2013 on the minimum health and safety requirements regarding the exposure of workers to the risks arising from physical agents (electromagnetic fields) and repealing Directive 2004/40/EC.

[10] EC. Directive 1997/43/Euratom of 30 June 1997 on health protection of individuals against the dangers of ionizing radiation in relation to medical exposure.

[11] EC. Directive 1996/29/Euratom of 13 May 1996 laying down basic safety standards for the protection of the health of workers and the general public against the dangers arising from ionizing radiation.

[12] EC. Directive 2006/25/EC of the European parliament and of the council of 5 April 2006 on the minimum health and safety requirements regarding the exposure of workers to risks arising from physical agents (artificial optical radiation).

[13] EC. Directive 2002/44/EC of 25 June 2002 on the minimum health and safety requirements regarding the exposure of workers to the risks arising from physical agents (vibration).

[14] EC. Directive 1989/686/EEC of 21 December 1989 on the approximation of the laws of the member states relating to personal protective equipment.

[15] Legido-Quigley H, McKee M, Nolte E, Glinos IA. Assuring the quality of health care in the European Union. Observatory Studies Series No 12; 2008., http:// www.euro.who.int/_data/assets/pdf_file/0007/98233/E91397.pdf.

[16] European Commission. DG health and consumer protection. Luxembourg declaration on patient safety; 2005. http://www.eu2005.lu/en/actualites/ documents_travail/2005/04/06Patientsafety/Luxembourg_Declaration_on_ Patient_Safety_05042005-1.pdf.

[17] European Commission. WHITE PAPER. Together for health: a strategic approach for the EU 2008-2013. COM(2007) 630 final; 2007., http://eur-lex. europa.eu/LexUriServ/site/en/com/2007/com2007_0630en01.pdf.

[18] CPME. Stakeholders' position paper on patient safety. Standing Committee of European Doctors; 2005. http://www.ehtel.org/forum/tasks-sources/tfpatient-safety-emedication/stakeholders_position_on_patient_safety.pdf.

[19] IAEA. Clinical training of medical physicists specializing in radiation oncology. Training course series, 37. International Atomic Energy Agency; 2009. http:// www-pub.iaea.org/MTCD/publications/PDF/TCS-37_web.pdf.

[20] IAEA. Clinical training of medical physicists specializing in diagnostic radiology. Training course series, 47. International Atomic Energy Agency; 2010. http://www-pub.iaea.org/MTCD/publications/PDF/TCS-47_web.pdf.

[21] IAEA. Clinical training of medical physicists specializing in nuclear medicine. Training course series, 50. International Atomic Energy Agency; 2011. http:// www-pub.iaea.org/MTCD/publications/PDF/TCS-50_web.pdf.

[22] EC Tuning Project. In: González J, Wagenaar R, editors. Tuning - Universities' contribution to the Bologna process. 2nd ed. Publicaciones de la Universidad de Deusto; 2008. http://www.unideusto.org/tuningeu/images/stories/ Publications/ENGLISH BROCHURE FOR WEBSITE.pdf.

[23] Tuning Physics Subject Area Group. Third cycle studies in physics; 2007. http://www.unideusto.org/tuningeu/tuning-3rd-cycle/physics.html.

[24] EFOMP Board, Caruana CJ. An initial EFOMP position on the tuning process for Masters Programs in Medical Physics in Europe. In: Dössel O, Schlegel WC, editors. WC 2009, IFMBE Proceedings 25/XIII; 2009. pp. 70-3.

[25] Caruana CJ. The tuning process and the Masters in Medical Physics in Europe - a personal vision. In: Tabakov S, editor. Medical Physics and Engineering Education and Training. Italy: ICTP Press; 2011. ISBN 92-95003. 